\title{
Apparel STEP Translator
}

Howard T. Moncarz Y. Tina Lee

U.S. DEPARTMENT OF COMMERCE National Institute of

Standards and Technology

Manufacturing Engineering Laboratory

Factory Automation Systems Division

Machine Intelligence Group

Gaithersburg, MD 20899-0001

June 1991

U.S. DEPARTMENT OF COMMERCE Robert A. Mosbacher, Secretary

NATIONAL INSTITUTE OF STANDARDS AND TECHNOLOGY

John W. Lyons, Director

$\begin{array}{ll}-Q C \longrightarrow & \text { red by } \\ 100 & \text { 1se Logistics Agency }\end{array}$

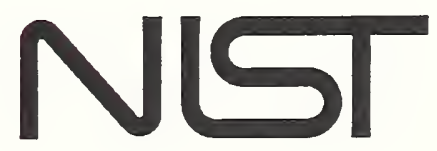

.056

\#4612 



\title{
Apparel STEP Translator
}

\author{
Howard T. Moncarz \\ Y. Tina Lee
}

U.S. DEPARTMENT OF COMMERCE National Institute of Standards and Technology Manufacturing Engineering Laboratory Factory Automation Systems Division Machine Intelligence Group Gaithersburg, MD 20899-0001

June 1991

U.S. DEPARTMENT OF COMMERCE Robert A. Mosbacher, Secretary

NATIONAL INSTITUTE OF STANDARDS AND TECHNOLOGY

JohnW. Lyons, Director

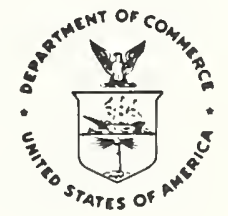




\section{Disclaimer}

Certain commercial equipment, instruments, or materials are identified in this paper in order to facilitate understanding. Such identification does not imply recommendation or endorsement by the National Institute of Standards and Technology, nor does it imply that the materials or equipment identified are necessarily the best available for the purpose. 


\title{
Apparel STEP Translator
}

\author{
by \\ Howard T. Moncarz \\ Y. Tina Lee \\ National Institute of Standards and Technology \\ June, 1991
}

\section{Abstract}

This paper describes the implementation of a prototype computer program for translating apparel pattern data between different file storage formats. The program demonstrates the feasibility of using a single, standard format as a mechanism for exchanging data between dissimilar pattern design systems. The program is an implementation of an information model previously developed at the National Institute of Standards and Technology (NIST) for apparel pattern data.

The software was developed as part of an ongoing project at NIST to incorporate apparel applications into the Standard for the Exchange of Product Model Data (STEP), an emerging international standard. The project is sponsored by the Defense Logistics Agency and the work is being carried out in cooperation with the Computer Integrated Manufacturing Committee of the American Apparel Manufacturers Association (AAMA). In the short term, the project goal is to develop a neutral data format for exchanging twodimensional pattern data between apparel CAD systems. In the longer term, the goal is to develop an information model that can be used to encompass the entire apparel life cycle. This achievement would allow all of the processes involved in the apparel life cycle to share the same data.

\section{Background}

The introduction of computers into the apparel industry has provided the potential for greater productivity, enhanced quality, and, in particular, faster turn-around time from design to manufacture. These goals have been achieved to some extent, but a bottle-neck occurs because the data produced by one manufacturing process (e.g. three-dimensional design) may be incompatible with that used by another (e.g. two-dimensional pattern making). This means, for example, that pattern data may not be easily exchanged between different manufacturing processes within the same factory. Furthermore, the data used and produced by the same manufacturing process implemented 
by two different CAD/CAM systems may also be incompatible. Consequently, there is no standard way of specifying pattern data that can be communicated unambiguously from one company to another.

The solution applied to this problem in the apparel industry, as well as in other industries, is to provide two-way translators for going from one specific data format to another. A separate translator is required for translation from every format to every other format and back again. Since there is a multitude of proprietary formats used to represent a pattern within the apparel industry, a proliferation of translators is the result. If any format is modified, all the translators going to or from that format must be modified.

An alternative approach is to specify a "neutral" data format as a single standard for data exchange. This permits each CAD/CAM producer to write only two translators, one going from their proprietary format to the neutral data format, and the other translator going the reverse direction. If any company changes its proprietary format, it must only modify its two translators, and no other company needs to be concerned. This paper describes a pattern data translator that uses this approach.

In November 1989, the task "Developing a Neutral Data Format for the Representation of 2D Pattern Pieces for the Apparel Industry" was assigned to the University of Southwestern Louisiana (USL) and the National Institute of Standards and Technology (NIST) by the AAMA Computer Integrated Manufacturing Committee (CIM/COM). As a result, it was recommended to use the DXF ${ }^{\mathrm{TM}}$ file format from AutoDesk, Inc. as a framework to develop a near-term neutral data format for pattern data. In addition, it was recommended to develop a STEP application model for the apparel industry as a long-term strategy. (STEP is the abbreviation for Standard for the Exchange of Product Model Data, an emerging international standard.) These two conclusions were presented to CIM/COM at the January, 1990 CIM/COM meeting, and were duly accepted by the members. The neutral data format for the translator described in this paper is based on the STEP approach. This was done to demonstrate the feasibility of using STEP in the apparel industry as the long-term strategy.

The goal of STEP is to develop a complete computer definition of the physical and functional characteristics of a product throughout that product's life cycle. As a standard, STEP will permit communications among computer environments, each of which performs various product life cycle functions. Initially, STEP will be used to exchange information between systems. Eventually, it will be used to integrate systems, using a shared database as the principal technique to do so.

In addition to the international effort, the U.S. is engaged in a domestic effort called Product Data Exchange using STEP (PDES). The goal of PDES is to develop the technology needed to implement the STEP objectives. In 1988 the National PDES Testbed was established at NIST to support this effort [McLean90]. Also in 1988, several major U.S. technology companies incorporated as PDES, Inc. with the specific goal of accelerating the development and implementation of STEP [Furlani90]. Among the projects being undertaken by PDES, Inc. at this testbed are the development of Application Protocols. An Application Protocol contains the information that is context sensitive to a particular application domain, and is the method by which the requirements for that domain are incorporated into STEP [Palmer91].

The ultimate goal for the work being done at NIST for the apparel industry is to develop one or more Application Protocols for apparel products. The immediate goal is to formulate a specification for apparel data exchange that borrows as much technology and conventions as possible from the PDES effort. In line with this immediate goal, an NIST apparel information model for representing two-dimensional pattern data [Lee90] has been formulated and is represented in the Express information modeling language. Express is a useful language for representing information models, and it is being used in the PDES effort to integrate different application domains into the 
STEP standard. Once the apparel information model has been developed and represented in Express, it can be used to map a particular two-dimensional pattern to a physical file in the STEP file format. A STEP physical file contains instances of the various entities defined by the Express information model.

\section{Design and Implementation}

To develop the specification for the neutral format and to demonstrate its usefulness, a computer program has been developed that will allow the exchange of two-dimensional pattern data between different industry formats. The translator uses a set of data structures that are derived from the NIST apparel information model mentioned above. This set of data structures, residing in computer memory, is referred to as the "working form." Data can be translated back and forth between different formats, using the working form as an intermediate buffer for that data. (See Figure 1.)

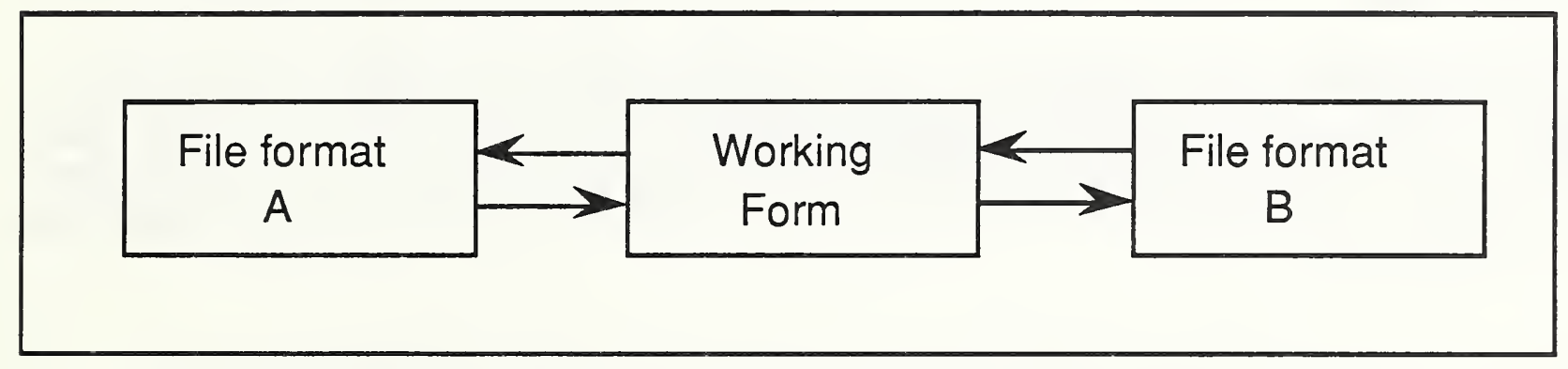

Figure 1. Translating between formats A \& B: the working form is used as an intermediate buffer.

The translator described here has been implemented for two industry formats: PN, a proprietary format from Gerber Garment Technology, Inc.; and DXF, from AutoDesk, Inc., used in their AutoCAD $\otimes$ product. (Other industry formats exist, but two are enough to illustrate the basic idea of this translator.) The program also supports a STEP physical file format derived from the NIST apparel pattern information model.

Each translation, either from industry format to working form or the reverse, is a two step process. To translate from industry format, the program first reads the data file and translates it to data structures that are derived from the industry file format. Next, those data structures are translated to the working form data structures that are derived from the Express model. For the reverse, to translate to industry format, the working form data structures are first translated to the data structures based on the industry format being generated. Then, in the second step, those data structures are used to generate the industry formatted file. (See Figure 2.) 


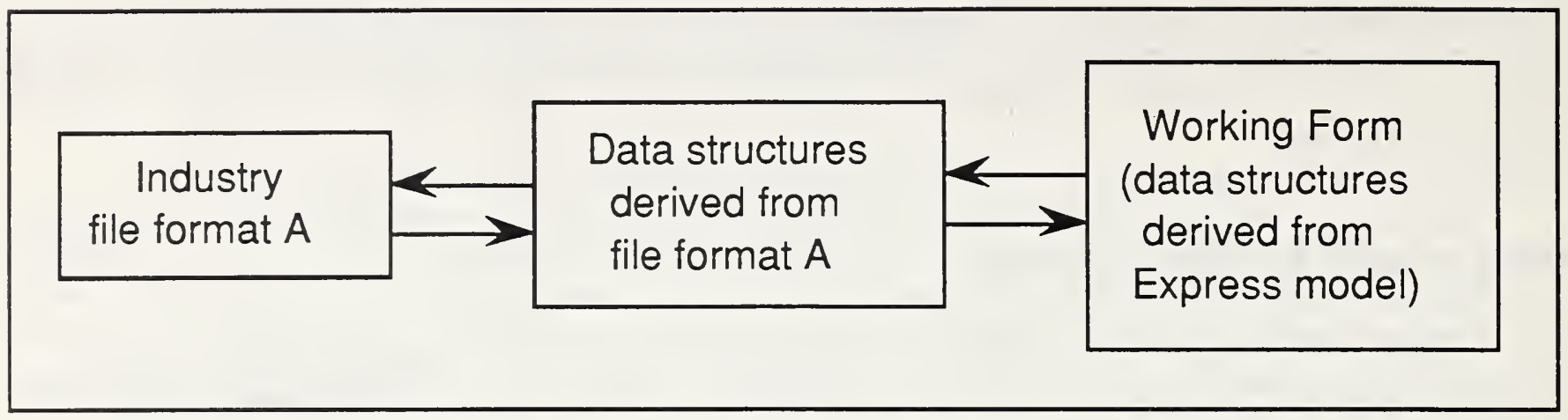

Figure 2. Two-step translation between the Working Form and File format $\mathrm{A}$

The file formats currently supported are text files. The translator allows the user to browse, edit and print these.

Once the data has been converted to working form, functions are available to the user to operate on that data. These functions include generating a two-dimensional pattern file in a different format, including the STEP format; displaying the pattern geometry on the screen; and printing the pattern geometry. (See Figure 3.) Other functions may be added by writing program modules that use the working form.

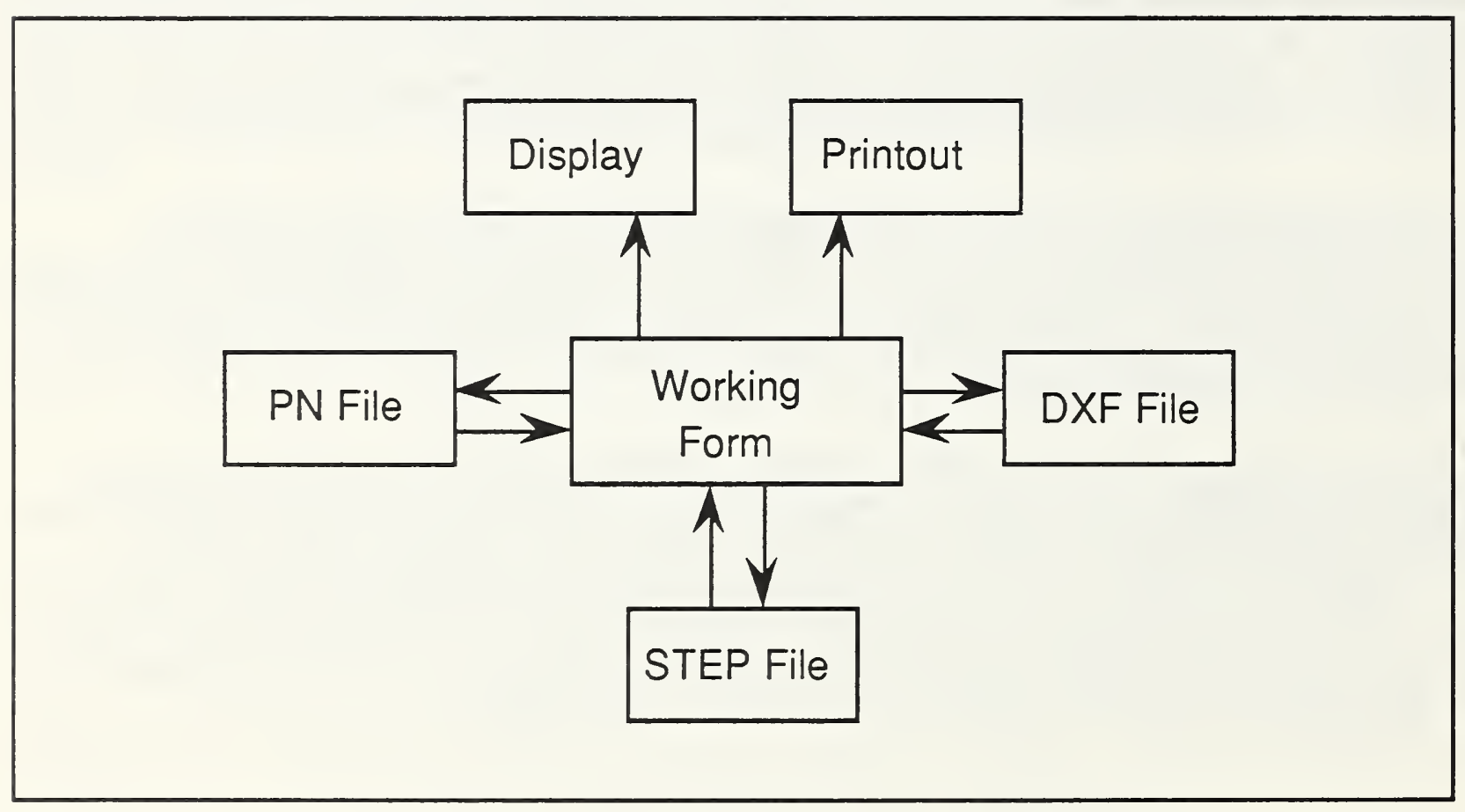

Figure 3. Functionality implemented

A graphical representation of a sample pattern is shown in the Appendix, along with the text file representation of that pattern in each of the three formats supported--PN, DXF, and STEP. The DXF and STEP file representations of the sample pattern would take up quite a few pages, if they were shown complete. Therefore, pieces of those files have been omitted in the appendix, indicated 
by the ellipses (...). Enough of the files are shown to give the reader a good idea of what the files would look like in their complete representation.

\section{User Interface}

The user interacts with the program through a graphics screen designed as a collection of windows. A window is a rectangular region on the screen that can be thought of as a separate terminal. Graphics and text can be displayed in the window. The user enters information to a window through a keyboard and a pointing device called a mouse. A button on the mouse allows the user to select an item by "clicking" it. Multiple windows can be used at the same time. This allows the user to look at the display in one window, while working with data in a different window.

When a session is started, the screen appears as shown in Figure 4. A main menu bar is shown along the top of the screen, and a window with the title, "2D Pattern Translator," is shown in the upper right region of the screen.

File Edit Parse Generate Special

Figure 4. Initial Screen 
The window is used to display a pattern's geometry, including its grading evolutions (or nests). The menu bar shows the groups of functions available to the user to operate the program. Any item in the menu bar may be selected, using the mouse, to reveal a list of functions associated with that item. Each of these lists is displayed in a vertical column below that item in the menu bar. Figure 5 shows all of the menu items that may be selected. (During operation, the user can only see one of these menus at a time.)

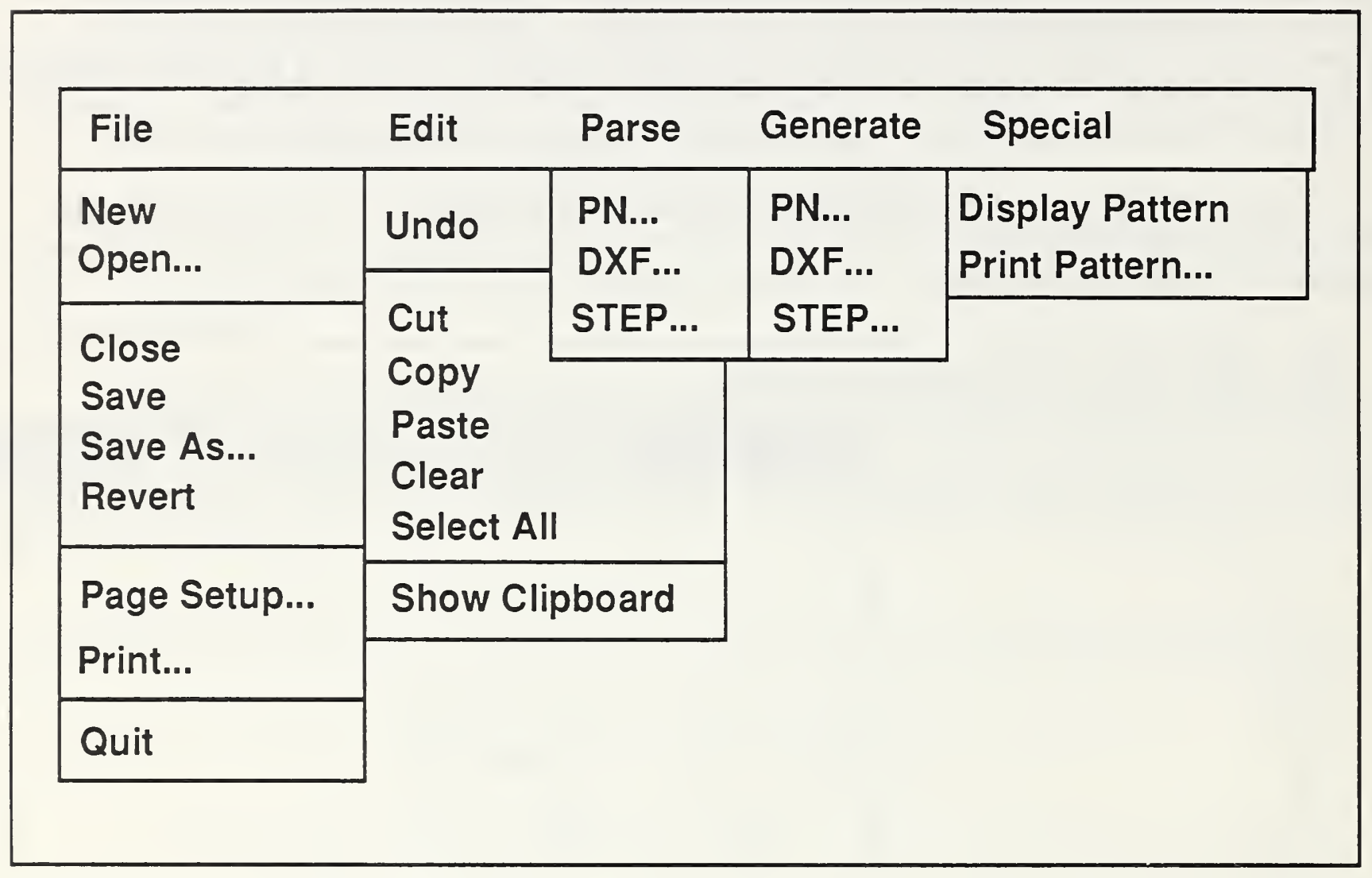

Figure 5. Menu bar with all items pulled down

The first two menus in the menu bar, File and Edit, are used to create and edit the text files that are read or generated by the program. When the user clicks the "Open" item in the File menu, a file requestor appears. A file requestor is a special type of window that allows the user to specify the name of a file to select. The ellipses (...) after "Open" in the menu item indicate that additional information will be required after this item is selected, before any action is invoked. In this case, that additional information is prompted by the file requestor. Once the file has been retrieved, a text window appears, and the text representation of the file is displayed in it. Multiple text windows can be opened. Each window permits a view into a file and allows the user to modify that file, using functions in the Edit menu. The items in the File menu, "Close" through "Revert," control whether the changes are stored permanently in the file or discarded.

The third menu, Parse, asks the user what file format to read, and then requests that the user enter the name of a file. The file is expected to be in the format selected. The program then attempts to read the file in the specified format and translate it to working form data structures in memory. If the file is successfully read and translated, a nest of the pattern will be displayed in the 2D Pattern Translator window. If there are errors, the user will be alerted, and error messages will be saved in an error file. The error file will appear automatically for the user to browse. The user can then call up another window to look at the erroneous data file with the corresponding error window indicating 
where and what the errors are. The data file can then be modified on-line and parsed again if desired.

The fourth menu, Generate, is the reverse of the Parse menu. This menu allows the user to translate the working form to one of the supported file formats.

The last menu, Special, contains functions that operate on the working form. Currently, a display function for refreshing the screen's display of the pattern piece and a print function for transferring that display to a printer are supported.

\section{Discussion}

The development of the Apparel STEP Translator Program was part of an ongoing effort to support the apparel industry in the development of standards for the apparel manufacturing life cycle. The AAMA has decided to use the AutoCAD DXF format as a starting point for an interim pattern data exchange standard, based on a report from the University of Southwestern Louisiana [USL90]. This standard will be a short term solution for exchanging pattern data. (As mentioned previously, STEP will be used as the long term solution.) Conventions (permitted within the DXF format) will be defined in the AAMA interim standard to support pattern data. The translator currently supports the DXF standard with a preliminary version of the conventions that are now being established. The translator will be modified to support the DXF standard as it evolves, and will be available to all parties involved in developing the standard. This will be useful in the evaluation of the standard and should help in its development.

One type of problem that must be addressed for any standard for pattern data exchange concerns the inconsistencies that exist among the different industry formats. In some cases, an entity defined in one industry format will have no analogous entity in another format. For example, there is one entity in the Gerber PN format called the "special piece number." This entity is used within a Gerber system, but has no relevance to systems made by other companies. Continued development of the translator program can uncover these types of problems. However, each company that has developed its own format is in the best position to implement that format as a module in the translator program. Such an exercise would be valuable in discovering potential problems with the standard being developed. Consequently, it is in everyone's interest to have the active support of as many companies as possible in developing the standard. Furthermore, development of commercial-grade applications using the standard (such as translator programs) is beyond the scope of NIST work and must be done by commercial parties.

Using STEP technology can leverage the apparel industry's efforts in three ways. The first is that modifications made to the apparel information model can be easily propagated to computer programs developed to use that model. This is because the information model itself is represented as data. The second advantage is that once the apparel model is incorporated into the STEP framework, all generic applications that have been developed for STEP models are available to be used with the apparel model. For example, the STEP file produced may be browsed and edited using programs developed for a generic STEP model. Finally, the third advantage is that using STEP technology will ensure that the standards developed by the U.S. apparel industry are consistent with international standards. This will encourage acceptance for U.S.products abroad.

The NIST PDES Toolkit [Clark90] is an example of STEP technology that is useful to any industry using STEP. This Toolkit is a set of software tools that is helpful for building applications that use STEP. The PDES Toolkit has been used to build a number of applications in other industries, but it has not yet been used in the apparel industry. At the time this project was begun, 
the PDES Toolkit had not been ported to the translator's computer platform. The tools are now being ported to this environment by staff from the National PDES Testbed.

The Apparel STEP Translator Program that has been implemented thus far has been developed from scratch, without the aid of the PDES Toolkit. The architecture of this program is similar to that which would be implemented using the toolkit, with some notable differences. The most important difference is that, for the current program, the information model is embedded in the system, rather than read in as data. This makes the program more difficult to maintain if the information model is modified. Furthermore, the features available to the user are those programmed for this particular application, and do not include any generic PDES functions that would be available if the toolkit were used. Once the PDES Toolkit has been ported to the translator's computer environment, the toolkit will be integrated into this computer program, and the benefits mentioned above can be accomplished.

\section{Concluding Remarks}

The computer program developed demonstrates the feasibility of using STEP technology in the apparel industry. The information model used was not intended to be a standard. It was developed for demonstration purposes. It did not include the consensus process necessary for developing a standard.

In addition to demonstration purposes, the flexibility of the program enables it to be used as a tool for standards development. The authors hope that this tool, and others like it, will encourage the use of STEP in the apparel industry.

NIST is now moving toward a more formal and active standards-development effort in the apparel PDES project. Under the Defense Logistics Agency's sponsorship, NIST will continue to work with AAMA to extend STEP to include apparel product data. The work will also be carried out with support from, and in cooperation with, standards organizations, the apparel manufacturing industry, and academia. 


\section{References}

[Clark90]

[Furlani90]

[Lee90]

[McLean90]

[Palmer91]

[USL90]
Clark, S. N., An Introduction to the NIST PDES Toolkit, NISTIR 4336, National Institute of Standards and Technology, Gaithersburg, MD, May 1990.

Furlani, C., et al., Status of PDES-Related Activities (Standards \& Testing), NISTIR 4432, National Institute of Standards and Technology, Gaithersburg, MD, October 1990.

Lee, Y. T., On Extending the Standard for the Exchange of Product Data to Represent Two-Dimensional Apparel Pattern Pieces, NISTIR 4358, National Institute of Standards and Technology, Gaithersburg, MD, June 1990.

McLean, C. R., National PDES Testbed Strategic Plan 1990, NISTIR 4438, National Institute of Standards and Technology, Gaithersburg, MD, October 1990.

Palmer, M., et al., Guidelines for the Development and Approval of STEP Application Protocols, ISO TC184/SC4/WG4 Document N1 Version 0.7 (Working Draft), ISO, January 1991.

Efe, Kemal, et al., Evaluation of Neutral Data Formats for the Representation of 2-D Pattern Pieces, USL A-CIM Technical Report \#2, University of Southwestern Louisiana, Lafayette, LA, February 1990. 
Appendix Sample Pattern Data

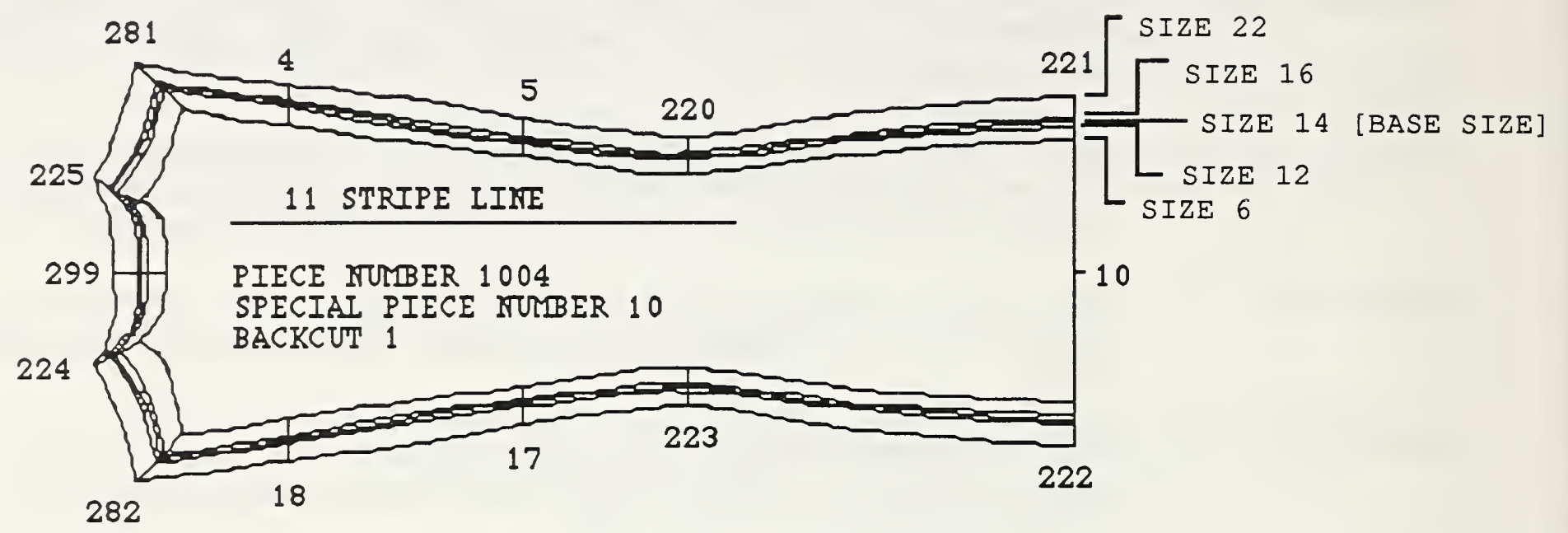

Nest of "Pattern Piece 1004" for base size (14) and four graded sizes $(6,12,16,22)$ 


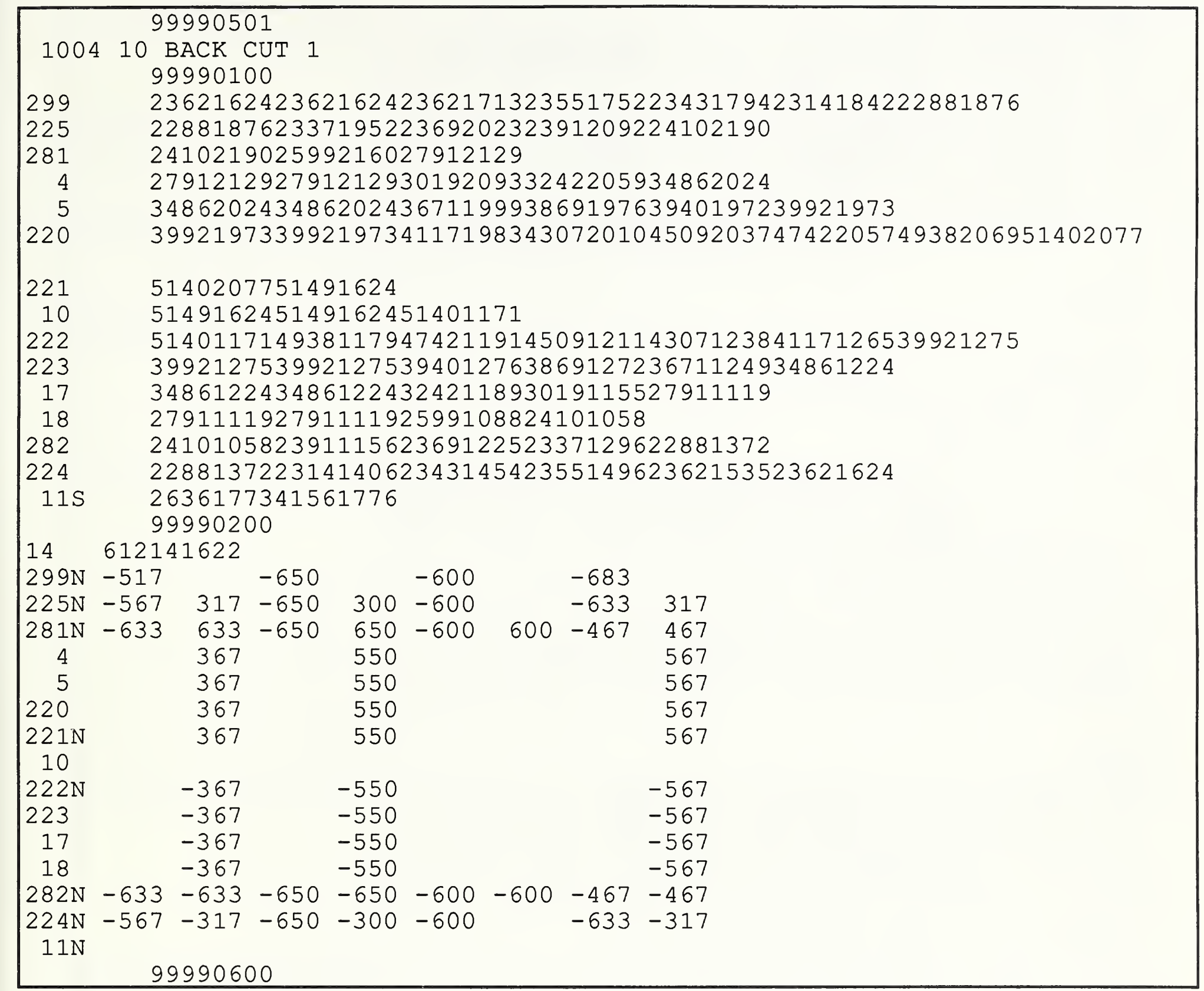

Gerber PN file for Pattern Piece 1004 
/* The file structure of this APDES STEP file is based on the Document 284 of ISO TC184/SC4/WG1oct. 27, 1988. This file has been presented in a line-oriented or record-oriented manner in order to aid readability. Spaces have also been added to aid readability. Note that an ordinary STEP file is not aligned in this manner, but is instead a continuous stream of characters.

*

STEP ;

HEADER ;

FILE IDENTIFICATION

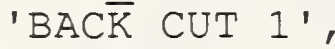

'19900816.091124',

('Tina Lee \& Howard Moncarz, (301) 975-3550 or 3350'),

('National Institute of Standards and Technology,

Factory Automation Systems Division'),

'APIM STEP VERSION 1.0',

'Preprocessor Version 1.0',

'Originating System - Gerber AM-5');

FILE DESCRIPTION (

'This file contains a sample APDES file');

IMP_LEVEL('1.0');

END $\bar{S} E C$;

DATA;

(299=TWO_D_COORDINATE $(23.62,16.24)$;

@225=TWO_D_COORDINATE $(22.88,18.76)$;

e281=TWO_D COORDINATE $(24.10,21.90)$;

@ 4=TWO_D_COORDINATE $(27.91,21.29)$;

$@ 11=$ TWO $\bar{D}$ COORDINATE $(26.36,17.73)$;

@ $1001=T \bar{W} O-D$ COORDINATE $(23.62,17.13)$;

@1002=TWO_D_COORDINATE $(23.55,17.52)$;

@ $1003=$ TWO_D_COORDINATE $(23.43,17.94)$;

Q1004=TWO D COORDINATE $(23.14,18.42)$;

@1037=TWO_D_COORDINATE $(41.56,17.76)$;

...

@1041=GRADE_POINTS (\#299,\#225,\#281,\#4, ..) ;

@1042=MARK_EEATURE (.NOTCH.,\#299);

@1043=MARK_EEATURE (.NOTCH . , \#4);

\section{Apparel STEP file for Pattern Piece 1004}

Note: For brevity, this is only a partial file. Enough of the file is shown to give the reader an idea of its format. The ellipses (...) indicate where portions of the file are omitted. The file is continued on the next page. 


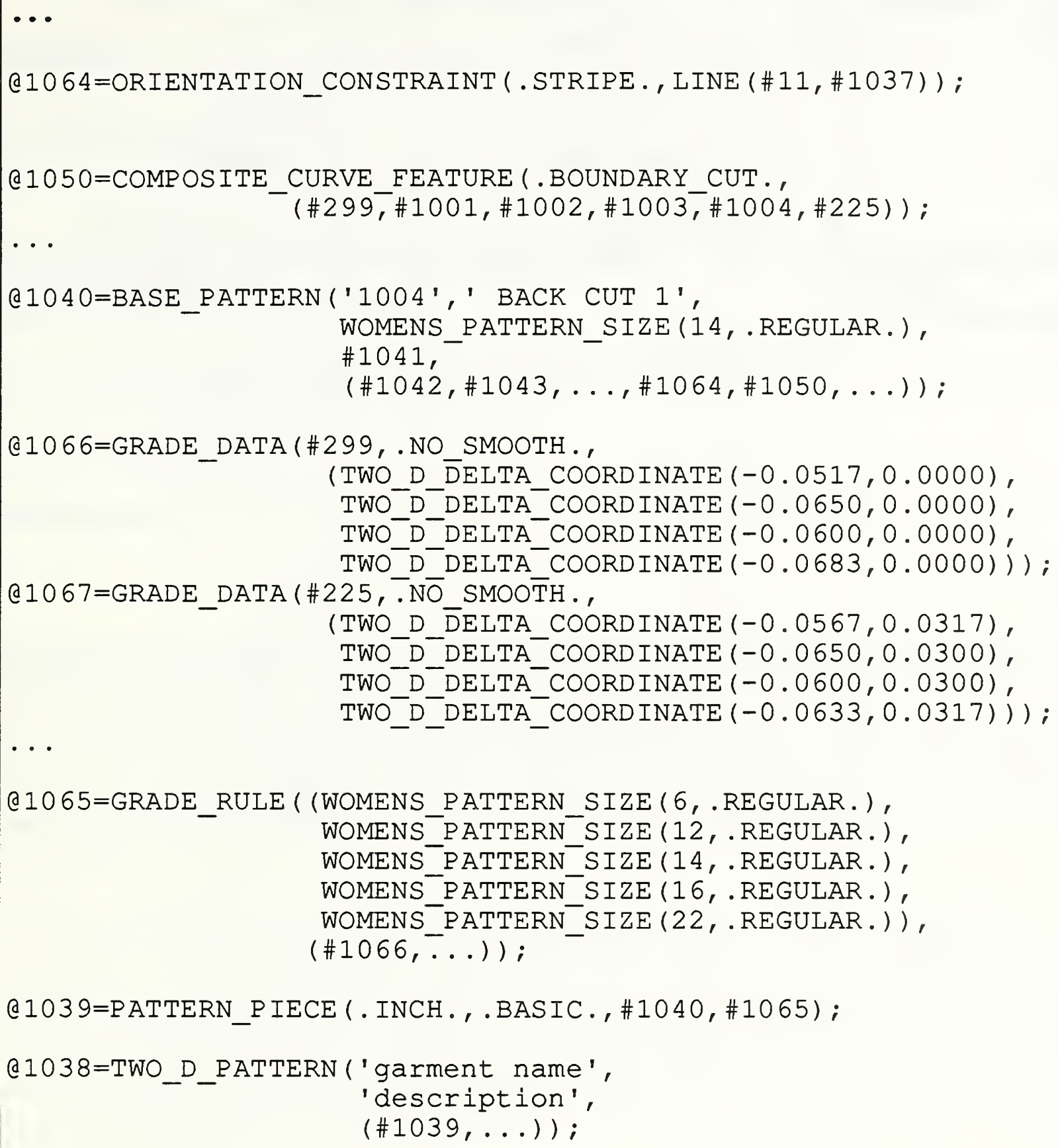

ENDSEC ;

ENDSTEP ;

\section{Apparel STEP file for Pattern Piecé 1004 (continued)}




\begin{tabular}{|c|c|c|}
\hline $\begin{array}{c}0 \\
\text { SECTION } \\
2 \\
\text { HEADER } \\
0 \\
\text { ENDSEC } \\
0 \\
\text { SECTION } \\
2 \\
\text { TABLES } \\
0 \\
\text { ENDSEC } \\
0 \\
\text { SECTION } \\
2 \\
\text { BLOCKS } \\
0 \\
\text { BLOCK } \\
8 \\
0 \quad \\
2 \\
\text { PIECEINFO } \\
70 \\
10 \\
0 \\
20 \\
0.00000 \\
0 \\
\text { TEXT }\end{array}$ & 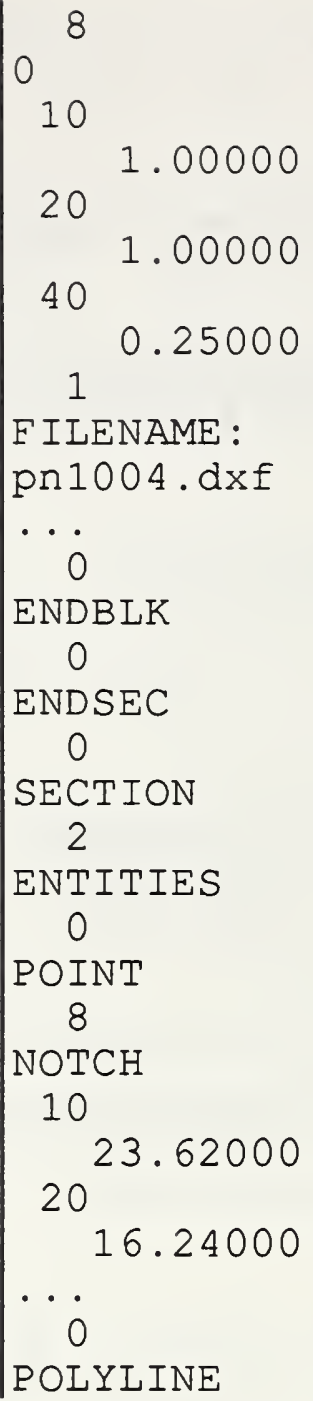 & $\begin{array}{l}8 \\
\text { BOUNDCUT } \\
66 \\
1 \\
0 \\
\text { VERTEX } \\
8 \\
\text { BOUNDCUT } \\
10 \\
23.62000 \\
20 \\
16.24000 \\
0 \\
\text { VERTEX } \\
8 \\
\text { BOUNDCUT } \\
10 \\
23.62000 \\
20 \\
17.13000 \\
\ldots \\
0 \\
\text { SEQEND } \\
0 \\
\text { POLYLINE } \\
\ldots \\
0 \\
\text { ENDSEC } \\
0 \\
\text { EOE }\end{array}$ \\
\hline
\end{tabular}

\section{AutoCAD DXF file for Pattern Piece 1004}

Note: For brevity, this is only a partial file. Enough of the file is shown to give the reader an idea of its format. The ellipses (...) indicate where portions of the file are omitted. The three columns of this file actually represent separate pages. 
NIST-1144

(REV. 3-00;
U.S. DEPARTMENT OF COMMERCE

NATIONAL INSTITUTE OF STANDARDS AND TECHNOLOGY

BIBLIOGRAPHIC DATA SHEET
1. PUDUCATIOH OR REPORT MUIDER

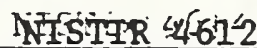

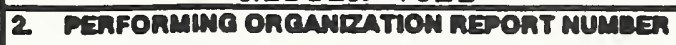

2. MULLIGATION DATE JUNE 1991

4. TIRE AND BUDTIUE

Apparel STEP Translator

5. AUTHOR(S)

Howard T. Moncarz, Y. Tina Lee

6. PERFORMIMO ORGAMIZATION (IF JOINT OR OTHER THAN MIST, SEE IMSTRUCTIOMS)

U.S. DEPANTMENT OF COMMERCE

MATIOMAL IMSTITUTE OF STANDANDS AND TECHMOLOCY

QATHERSEURA, MO 20000

7. CONTMCT/GRANT MUMBER

91-R\&D-1

Q. TYPE OF REPOAT AND PERIOD COVERED

9. SPOMSORIMQ ORQANIZATIOM MAME AND COMPLETE ADDRESS (STREET, CTT, STATE, ZIT)

Defense Logistics Agency

Manufacturing Engineeering/Research Office

Cameron Station-DLA-PR

Alexandria, VA 22304-6100

10. SUPPLEMENTARY MOTES

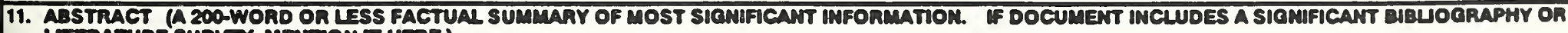
UTERATURE SUAVE, MEMTION IT HERE)

This paper describes the implementation of a prototype computer program for translating apparel pattern data between different file storage formats. The program demonstrates the feasibility of using a single, standard format as a mechanism for exchanging data between dissimilar pattern design systems. The program is an implementation of an information model previously developed at National Insitute of Standards and Technology (NIST) for apparel pattern data.

The software was developed as part of an ongoing project at NIST to extend the emerging international standard, Standard for the Exchange of Product Model Data (STEP), to apparel applications. The project is sponsored by the Defense Logistics Agency and the work is being carried out in cooperation with the Computer Integrated Manufacturing committee of the American Apparel Manufacturers Association (AAMA). In the short term, the project goal is to develop a neutral data format for exchanging two-dimensional pattern data between apparel CAD systems. In the longer term the goal is to develop an information model that can be used to encompass the entire apparel life cycle. This achievement would allow all the processes Involved in the apparel ife cycle to share the same data.

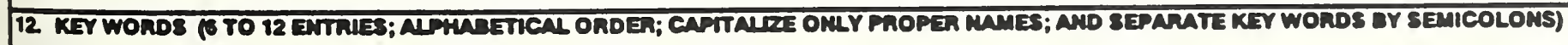
apparel; APDES; APIM; CAD/CAM; data exchange; DXF; neutral file format; pattern; PN; product data; PDES; STEP

\begin{tabular}{|l}
\hline \\
\hline
\end{tabular}

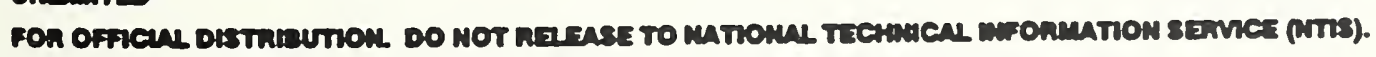

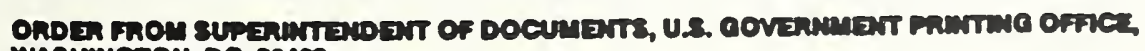
WASHavetom, DC sonce 


